\title{
Management of a Case of Hypersplenism for Therapeutic Splenectomy: Anesthesia Challenges
}

\author{
Fatema K Mujpurwala ${ }^{1}$, Pallavi A Kharat ${ }^{2}$, Naina Dalvi ${ }^{3}$, Jayesh Bawa ${ }^{4}$, Geeta Ghag ${ }^{5}$, Vipul Nandu ${ }^{6}$
}

\begin{abstract}
Hypersplenism is a clinical disorder characterized by splenic enlargement and overactivity adversely affecting a patient's hematological profile. ${ }^{1}$ Splenectomy is associated with numerous intraoperative and postoperative challenges to an anesthesiologist. A 60-year-old woman presented with pain in the abdomen and fever with physical findings of pallor, icteric sclera, and splenomegaly. She was planned for therapeutic splenectomy under general anesthesia. We present a case report of a successfully managed case of hypersplenism for splenectomy.

Keywords: Anesthesia challenges, Hypersplenism, Splenectomy, Thrombocytopenia.

Research and Innovation in Anesthesia (2021): 10.5005/jp-journals-10049-0112
\end{abstract}

\section{INTRODUCTION}

Hypersplenism is a clinical disorder characterized by splenic enlargement and overactivity affecting one or more hematological lineages. ${ }^{1}$ It adversely affects the patient's hematological profile leading to anemia, thrombocytopenia, and leukopenia. ${ }^{2}$ It occurs in many underlying conditions such as alcoholism, myeloid lymphoproliferative disorder, liver cirrhosis, and malignancies., ${ }^{1,2}$

Splenectomy is associated with numerous intraoperative and postoperative challenges to anesthesiologists including perioperative coagulation abnormalities, intraoperative massive hemorrhage, and post-splenectomy infections. ${ }^{3}$ While caring for the patients scheduled for splenectomy with hypersplenism, the anesthesia plan must address both patient and procedure-specific concerns. $^{3}$

\section{Case Description}

We report a case of a 60 -year-old woman weighing $52 \mathrm{~kg}$, with hypersplenism posted for therapeutic splenectomy. She presented with a history of on and off fever and abdominal fullness for 4 months. Fever was intermittent and low grade not associated with chills and rigors. There was no history of burning micturition. Her complaint of abdominal fullness progressed gradually which resulted in postprandial fullness and finally, she had a loss of appetite. She was admitted to a tertiary care center with these complaints and was found to have pancytopenia with hemoglobin of $6 \mathrm{~g} / \mathrm{dL}, \mathrm{WBC}$ of 3,100 , and platelet count of $54,000 / \mathrm{mm}^{3}$. Her bone marrow aspiration was done which showed hypercellular marrow. Her bone marrow biopsy was done which revealed hypercellular bone marrow with trilineage hematopoiesis showing erythroid hyperplasia, increased megakaryocyte, and myeloid series with a shift to left. CT abdomen revealed splenomegaly. Her PET-CT was done which showed hypermetabolic hepatosplenomegaly and increased metabolic uptake in bowel loops with few weakly metabolic peritoneal nodules. She became COVID positive in March 2021 during the course of hospitalization but did not require any oxygen support. She was transferred to our hospital for further management. On physical examination, she had pallor, icteric

\footnotetext{
${ }^{1-5}$ Department of Anesthesia, Hindu Hriday Samrat Balasaheb Thackeray Medical College and Dr Rustom Narsi Cooper Muncipal General Hospital, Mumbai, Maharashtra, India

${ }^{6}$ Department of Surgery, Shri Vasantrao Naik Government Medical College, Yavatmal, Maharashtra, India

Corresponding Author: Fatema K Mujpurwala, Department of Anesthesia, Hindu Hriday Samrat Balasaheb Thackeray Medical College and Dr Rustom Narsi Cooper Muncipal General Hospital, Mumbai, Maharashtra, India, Phone: +91 9512472387, e-mail: drfatemaanesthesia@gmail.com

How to cite this article: Mujpurwala FK, Kharat PA, Dalvi N, et al. Management of a Case of Hypersplenism for Therapeutic Splenectomy: Anesthesia Challenges. Res Inno in Anesth 2021;6(2):54-56.

Source of support: Nil

Conflict of interest: None
}

sclera, and gross splenomegaly up to the right iliac fossa. The rest of the systemic examination was normal. Her blood investigations revealed a hemoglobin level of $7.8 \mathrm{~g} \%$, white blood cell 3,100, platelet count $50,000 / \mathrm{mm}^{3}$, total bilirubin $1.7 \mathrm{mg} / \mathrm{dL}$, direct bilirubin $0.8 \mathrm{mg} / \mathrm{dL}$, prothrombin time (PT) and international normalized ratio (INR) $16 / 13$ and 1.22 , respectively. The rest of the liver function test, serum creatinine, and serum electrolytes were within normal limits. Despite all the investigations, no confirm diagnosis regarding splenomegaly could be made and she had persistent pancytopenia with decreasing platelet count. So, the decision of therapeutic splenectomy was taken in view of her deteriorating clinical condition. The patient was immunized with pneumococcal, meningococcal, and Haemophilus influenzae vaccines 2 weeks before surgery. The patient was hydrated with $1.5 \mathrm{~L}$ preoperatively. Injection vitamin K (10 mg, IV BID) was administered preoperatively for 2 days.

On the day of surgery, venous access was secured with wide bore $18 \mathrm{G}$ intravenous cannula and infusion of Ringer lactate was started. The patient was premedicated with injection glycopyrrolate $40 \mu \mathrm{g} / \mathrm{kg}$, injection midazolam $0.15 \mathrm{mg} / \mathrm{kg}$, and injection buprenorphine $90 \mu \mathrm{g}$ iv. After preoxygenation for 3 minutes, anesthesia was induced with inj. propofol $2 \mathrm{mg} / \mathrm{kg}$ 
and inj. succinylcholine $2 \mathrm{mg} / \mathrm{kg}$ iv and intubated with a $7.0 \mathrm{~mm}$ cuffed portex endotracheal tube. Anesthesia was maintained with oxygen and air 50:50 concentration, desflurane 5-6\% dial setting and inj. vecuronium. The left radial artery was cannulated with a 20G cannula after performing Allen's test and an ultrasound-guided right internal jugular vein was cannulated with a $7.0 \mathrm{~F}$ central venous catheter. The central venous pressure measure was around 8-10 $\mathrm{cm}$ of water. Hypothermia was avoided by infusing warm IV fluids, warm blood, and by using a body warmer. The surgery lasted for 4 hours and total intraoperative blood loss was around $2 \mathrm{~L}$ and urine output was $550 \mathrm{~mL}$. The patient received a total of 1,500 mL of crystalloids and was transfused 2 units of whole blood and 10 random donor platelets (RDP) after clamping of splenic vessels. The massively enlarged spleen was removed as shown below, it weighed $1.920 \mathrm{~kg}$ (Fig. 1).

Intraoperatively during vascular clamping inj. noradrenaline (8 $\mathrm{mg}$ in $50 \mathrm{~mL}$ ) was started @ $8 \mathrm{~mL} /$ hour as there was a sudden blood loss of $1 \mathrm{~L}$ followed by hypotension of $90 / 60 \mathrm{~mm} \mathrm{Hg}$. At the end of the surgery, local infiltration was given with $20 \mathrm{~mL}$ of $0.25 \%$ bupivacaine. The patient was not extubated in view of massive blood loss, thrombocytopenia, vasopressor support, and was electively ventilated and shifted on ventilatory support to our anesthesia ICU (AICU). Postoperatively patient received 4 units of fresh frozen plasma (FFP). The patient was extubated on a postoperative day 2 and inj. noradrenaline was slowly tapered off. Injection paracetamol $1 \mathrm{~g}$ iv qid, injection tramadol $50 \mathrm{mg}$ iv bd, and inj. diclofenac $75 \mathrm{mg}$ iv sos were used to manage postoperative pain. On postoperative day 2 , her hemoglobin was $11.6 \mathrm{~g} / \mathrm{dL}$, WBC count was 18,200 , and platelet count was $241,000 / \mathrm{mm}^{3}$. The postoperative course was simple without any postoperative complications. Her spleen histopathology report revealed dilated sinusoids of red pulp with extensive extramedullary hematopoiesis comprising of the trilineage proliferation of myeloid, lymphoid, and megakaryocytic series with a focus on wedge-shaped infarcts. The patient remained stable postoperatively and was discharged after 2 weeks.

\section{Discussion}

Hypersplenism is defined as a clinical disorder with characteristic splenic enlargement and overactivity affecting one or more hematological lineages. ${ }^{1}$ It adversely affects the patient's hematological profile leading to anemia, thrombocytopenia, and leukopenia. ${ }^{2}$ It leads to platelets sequestration of about $30 \%$ which can reach up to $90 \%{ }^{1}$ Hypersplenism can be secondary to liver cirrhosis, infections, myeloid lymphoproliferative disorder, malignancies, and alcoholism. Our patient had hypersplenism secondary to hematopoietic dysplasia, more likely to be malignant. Mostly primary splenic cancers are uncommonly encountered in the practice of general surgical pathology. Lymphomas like chronic lymphocytic leukemia, hairy cell leukemia, splenic marginal zone lymphoma, and myeloproliferative neoplasms like chronic myeloid leukemia, polycythemia vera, essential thrombocythemia, primary and secondary myelofibrosis are the most prevalent hematological malignancies associated with splenomegaly. ${ }^{4}$ Although neoplasms of the spleen are rare entities they pose a diagnostic challenge and are found incidentally after splenectomies. Few of the splenic neoplasms have malignant potency and make the spleen fragile, thereby their fast and accurate diagnosis is imperative. ${ }^{5}$

Splenectomy can be used to treat hypersplenism. Anesthetic management requires a multidisciplinary collaboration of anesthesiologists, surgeons, hematologists, and gastroenterologists. ${ }^{1}$ The anesthesia challenges include perioperative management of moderate to severe thrombocytopenia and associated hemostasis disorder which increases the risk of bleeding in case of invasive procedure. Intraoperative management of massive hemorrhage and postoperative complications like portal vein and mesenteric vein thrombosis (incidence $7-10 \%$ ) and asplenia predisposing patients to a higher risk of overwhelming post-splenectomy infection (OPSI). ${ }^{2}$ Vaccination before splenectomy is a must to prevent postoperative infections. ${ }^{6}$

Partial splenic embolization (PSE), a non-surgical intervention has emerged as a newer mode of treatment. Partial splenic embolization presents several advantages over conventional splenectomy which includes increased average speed of the procedure, early postprocedural ambulation, lack of need for blood transfusion, and preservation of splenic tissue function to protect against infections. ${ }^{2,7}$

\section{Conclusion}

We could successfully manage the case of hypersplenism for therapeutic splenectomy. Splenectomy for hypersplenism was effective with a good postoperative outcome. Anesthesia management includes proper perioperative management of thrombocytopenia and other hemostasis disorders, with drastic
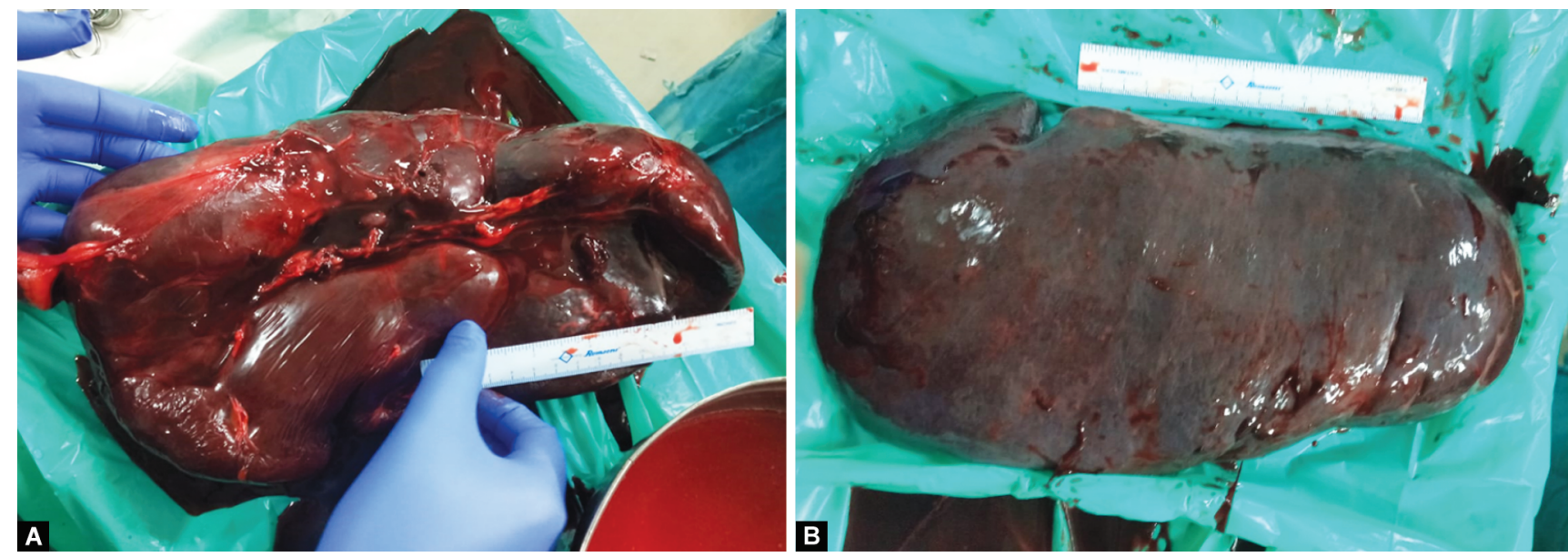

Figs 1 A and B: Splenectomy specimens 
measures for the management of intraoperative hemorrhagic shock. Vaccination before splenectomy is a must to prevent postoperative infections.

\section{References}

1. MI F, Getting anesthetic management of splenectomy for hypersplenism at the University Hospital of the Aristide Le Dantec hospital. 3.

2. Talwar A, Gabr A, Riaz A, et al. Adverse events related to partial splenic embolization for the treatment of hypersplenism: a systematic review. J Vasc Interv Radiol JVIR 2020;31(7):Available from: https:// pubmed.ncbi.nlm.nih.gov/32014400/.
3. Asnis J, Neustein SM. General anesthesia complicated by perioperative iatrogenic splenic rupture. Middle East J Anaesthesiol 2012;21(4):619-622.

4. Kienle DL. The spleen in hematologic malignancies. Ther Umsch Rev Ther 2013;70(3):163-169.

5. Fotiadis C, Georgopoulos I, Stoidis C, et al. Primary tumors of the spleen. Int J Biomed Sci IJBS 2009;5(2):85-91.

6. Chaithanya K, Reddy PN, Gandra S, et al. Anaesthetic management of a case of hereditary spherocytosis for splenectomy and cholecystectomy. Indian J Anaesth 2014;58(3):343-345.

7. Amin MA, El Gendy MM, Dawoud IE, et al. Partial splenic embolization versus splenectomy for the management of hypersplenism in cirrhotic patients. World J Surg 2009;33(8):1702-1710. 